\title{
A UNIT DISC ANALOGUE OF THE BANK-LAINE CONJECTURE DOES NOT HOLD
}

\author{
Janne Heittokangas and Kazuya Tohge \\ University of Eastern Finland, Department of Physics and Mathematics \\ P.O. Box 111, 80101 Joensuu, Finland; janne.heittokangas@uef.fi \\ Kanazawa University, College of Science and Engineering \\ Kakuma-machi, Kanazawa 920-1192, Japan; tohge@t.kanazawa-u.ac.jp
}

\begin{abstract}
The 1982 conjecture due to Bank and Laine claims the following: If $A(z)$ is a transcendental entire function of order of growth $\rho(A) \in[0, \infty) \backslash \mathbf{N}$, then $\max \left\{\lambda\left(f_{1}\right), \lambda\left(f_{2}\right)\right\}=\infty$, where $f_{1}, f_{2}$ are linearly independent solutions of $f^{\prime \prime}+A(z) f=0$ and $\lambda(g)$ stands for the exponent of convergence of the zeros of $g$. This conjecture has been verified in the case $\rho(A) \leq 1 / 2$, while counterexamples have been found in the cases $\rho(A) \in \mathbf{N} \cup\{\infty\}$. The aim of this paper is to illustrate that no growth condition on $A(z)$ alone yields a unit disc analogue of the Bank-Laine conjecture. The main discussion yields solutions to two open problems recently stated by Cao and Yi.
\end{abstract}

\section{Introduction}

The celebrated 1982 paper by Bank and Laine [3] opened up a new chapter in the oscillation theory of solutions of

$$
f^{\prime \prime}+A(z) f=0
$$

where $A(z)$ is entire [17]. Finding a growth condition on $A(z)$ such that every fundamental solution base $\left\{f_{1}, f_{2}\right\}$ of (1.1) satisfies

$$
\max \left\{\lambda\left(f_{1}\right), \lambda\left(f_{2}\right)\right\}=\infty
$$

has aroused wide interest during the last three decades. Here and in what follows,

$$
\lambda(g)=\inf \left\{\alpha>0: \sum_{n=1}^{\infty}\left|z_{n}\right|^{-\alpha}<\infty\right\}
$$

stands for the exponent of convergence of the zeros $\left\{z_{n}\right\}$ of an entire function $g$, while

$$
\rho(g)=\limsup _{r \rightarrow \infty} \frac{\log T(r, f)}{\log r}
$$

is the order of growth of $g$. If $A(z)$ is a polynomial, then it is well-known $[3,17]$ that all solutions $f$ of (1.1) are entire and satisfy $\lambda(f) \leq \rho(f)<\infty$. Hence, in all attempts to obtain (1.2), we need to assume that $A(z)$ is transcendental.

Bank and Laine [3] introduced a method for constructing equations of the form (1.1) with zero-free solution bases. This construction depends on a certain entire

doi:10.5186/aasfm.2011.3622

2010 Mathematics Subject Classification: Primary 34M10; Secondary 30D35.

Key words: Asymptotic growth, Bank-Laine conjecture, exponent of convergence, linear differential equation, Nevanlinna theory, oscillation, unit disc, zero-free solution base.

This research was supported in part by the Academy of Finland \#121281, and by the Japan Society for the Promotion of Science Grant-in-Aid for Scientific Research (C) \#19540173. 
parameter function $\varphi$. If $\varphi$ is a polynomial, then $\rho(A)$ is a positive integer, while if $\varphi$ is transcendental, then $\rho(A)=\infty$. Hence it seems plausible that (1.2) holds whenever $A(z)$ is transcendental and satisfies $\rho(A) \in[0, \infty) \backslash \mathbf{N}$. This is widely known as the Bank-Laine conjecture, which we abbreviate as the BL-conjecture.

To get an intuitive idea on the theory behind the BL-conjecture, we outline the general approach from [3]: Let $\left\{f_{1}, f_{2}\right\}$ be a fundamental solution base of (1.1) with Wronskian $W\left(f_{1}, f_{2}\right)=c \neq 0$. Denote $E=f_{1} f_{2}$. Then

$$
4 A(z)=\left(\frac{E^{\prime}}{E}\right)^{2}-\left(\frac{c}{E}\right)^{2}-2 \frac{E^{\prime \prime}}{E} .
$$

A standard Nevanlinna theory reasoning applied to (1.3) results in

$$
2 T(r, E)=2 \bar{N}\left(r, \frac{1}{E}\right)+T(r, A)+S(r, E) .
$$

Now, suppose that some growth condition on a finite-order entire $A(z)$ forces $\rho(E)=$ $\infty$. Then, by (1.4), it follows that $\lambda(E)=\infty$, which clearly yields (1.2).

The BL-conjecture was verified in $[3]$ in the case $\rho(A)<1 / 2$ by means of WimanValiron theory and the $\cos \pi \rho$-theorem. The case $\rho(A)=1 / 2$ was proved independently by Rossi [24] and Shen [27]. The method in [24] is based on the Beurling-Tsuji estimate for harmonic measure, while the method in [27] relies on the Carleman integral inequality. We note that the BL-conjecture still remains unsolved [18].

Proceeding to the case of the unit disc $\mathbf{D}$, we need the following definitions. Let $g$ be an analytic function in $\mathbf{D}$. The exponent of convergence of the zeros $\left\{z_{n}\right\}$ of $g$ is given by

$$
\mu(g)=\inf \left\{\beta>0: \sum_{n=1}^{\infty}\left(1-\left|z_{n}\right|\right)^{\beta+1}<\infty\right\},
$$

while the order of growth of $g$ is

$$
\sigma(g)=\limsup _{r \rightarrow 1^{-}} \frac{\log ^{+} T(r, g)}{-\log (1-r)}
$$

Recall that the inequality $\sigma(g) \geq \mu(g)$ always holds. If $g$ has the growth rate

$$
\limsup _{r \rightarrow 1^{-}} \frac{T(r, g)}{-\log (1-r)}=\infty
$$

then $g$ is called admissible. The Nevanlinna error term $S(r, g)$ is of growth $o(T(r, g))$, provided that $g$ is admissible. We say that $g$ belongs to the Korenblum space $A^{-\infty}$ [15] if there exists a constant $q \in[0, \infty)$ such that

$$
\sup _{z \in \mathbf{D}}\left(1-|z|^{2}\right)^{q}|g(z)|<\infty .
$$

Further, $g$ belongs to the Hardy space $H^{p}[9], p \in(0, \infty]$, if

$$
\begin{aligned}
\sup _{0 \leq r<1} \int_{0}^{2 \pi} \mid g\left(\left.r e^{i \theta}\right|^{p} d \theta<\infty,\right. & p<\infty, \\
\sup _{z \in \mathbf{D}}|g(z)|<\infty, & p=\infty .
\end{aligned}
$$

The harmonic counterpart of the $H^{p}$ space is denoted by $h^{p}[9]$.

The space $A^{-\infty}$ includes the classical $H^{p}$-spaces, for if $g \in H^{p}, 0<p \leq \infty$, then (1.5) holds for $q=1 / p\left[9\right.$, p. 36]. Moreover, if $g \in A^{-\infty}$, then $g$ is non-admissible, and 
so $\sigma(g)=0$. While discussing differential equations in the unit disc, the functions in the Korenblum space have sometimes been called $\mathscr{H}$-functions since the appearance of [12].

Next, let $A(z)$ be analytic in $\mathbf{D}$, and let $\left\{f_{1}, f_{2}\right\}$ be a fundamental solution base of (1.1). We wish to find a growth condition on $A(z)$ such that

$$
\max \left\{\mu\left(f_{1}\right), \mu\left(f_{2}\right)\right\}=\infty .
$$

This would be a unit disc analogue of the BL-conjecture. If $A(z)$ is an $\mathscr{H}$-function, then it is known that all solutions $f$ of $(1.1)$ satisfy $\mu(f) \leq \sigma(f)<\infty$ [12]. Hence, for (1.6) to hold, $A(z)$ cannot be an $\mathscr{H}$-function.

The aim of this paper is, however, to illustrate that no growth condition on $A(z)$ alone implies (1.6). This discussion is carried out in Section 2. The main result is Theorem 1 below, which will be proved in Section 4 . The proof requires that we compare the growth of characteristics $T(r, \varphi)$ and $T\left(r, e^{\varphi}\right)$ for a function $\varphi$ analytic in D. This comparison, to be presented in Section 3, is closely related to the well known paper by Clunie [8] in the case of the complex plane. The main discussion yields solutions to two open problems recently stated by Cao and $\mathrm{Yi}$ in [4], see Section 2. Concluding remarks on zero-free solution bases are given in Section 5.

\section{Construction of zero-free solution bases}

Following the reasoning in [3, p. 356], let $\varphi$ be an analytic function in $\mathbf{D}$, and let $h$ denote a primitive function of $e^{\varphi}$, that is, $h^{\prime}=e^{\varphi}$. Set $g=-(\varphi+h) / 2$. Then a simple computation shows that the functions $f_{1}=e^{g}$ and $f_{2}=e^{g+h}$ are linearly independent solutions of (1.1), where

$$
A=-\frac{1}{4}\left(e^{2 \varphi}+\left(\varphi^{\prime}\right)^{2}-2 \varphi^{\prime \prime}\right)
$$

is analytic in $\mathbf{D}$. Note that the choice $\varphi(z)=-\log (1-z)$ implies $A(z) \equiv 0$ and gives raise to linearly independent solutions $f_{1}(z)=1-z$ and $f_{2}(z)=1$ having no zeros in D. Suppose more generally that $\varphi$ belongs to the Bloch space $\mathscr{B}[1,22]$, that is,

$$
\|\varphi\|_{\mathscr{B}}=\sup _{z \in \mathbf{D}}\left(1-|z|^{2}\right)\left|\varphi^{\prime}(z)\right|<\infty .
$$

Then

$$
|\varphi(z)| \leq\|\varphi\|_{\mathscr{B}}\left(1+\frac{1}{2} \log \left(\frac{1+|z|}{1-|z|}\right)\right) \quad \text { and } \quad\left|\varphi^{(j)}(z)\right| \leq \frac{2^{4(j-1)}\|\varphi\|_{\mathscr{B}}}{\left(1-|z|^{2}\right)^{j}}
$$

where $z \in \mathbf{D}$ is arbitrary and $j=1,2$. A substitution to (2.1) now shows that $A(z)$ is an $\mathscr{H}$-function, and hence every solution of (1.1) is of finite order of growth [12]. In the following example we consider cases where $A(z)$ is not an $\mathscr{H}$-function.

Example 1. (1) The choice $\varphi(z)=\frac{1+z}{1-z}$ in (2.1) leads to a coefficient $A(z)$ growing exponentially, yet $A(z)$ is of bounded characteristic. Indeed,

$$
T\left(r, e^{2 \varphi}\right)=2 m\left(r, e^{\varphi}\right)=\frac{1}{\pi} \int_{0}^{2 \pi} \frac{1-|z|^{2}}{|1-z|^{2}} d \arg (z)=2, \quad 0<r=|z|<1,
$$

and therefore $e^{2 \varphi}$ is of bounded characteristic. Moreover, $\left(\varphi^{\prime}\right)^{2} \in H^{p}$ for all $p<1 / 4$ and $\varphi^{\prime \prime} \in H^{p}$ for all $p<1 / 3[9]$, and hence both $\left(\varphi^{\prime}\right)^{2}$ and $\varphi^{\prime \prime}$ are of bounded characteristic. 
(2) For $k \in \mathbf{N} \backslash\{1\}$ and $c \in(-1,0)$, let

$$
\psi(z)=\sum_{n=1}^{\infty} k^{c n} z^{k^{n}}
$$

be the function studied by Hayman [10] and Littlewood [20]. Since $\psi$ is bounded in $\overline{\mathbf{D}}$, it is analytic and of bounded characteristic in $\mathbf{D}$. If $k$ is large enough, then $\psi^{\prime}$ is of unbounded characteristic. However, a calculation based on Cauchy's integral formula shows that

$$
\sup _{z \in \mathbf{D}}\left(1-|z|^{2}\right)^{j}\left|\psi^{(j)}(z)\right|<\infty
$$

for all $j \in \mathbf{N}$. Choose $\varphi=\psi^{\prime}$, and suppose on the contrary that $e^{\varphi}$ is of bounded characteristic. By [11, p. 174] we have $\Re \varphi \in h^{1}$. Kolmogorov's theorem [9, p. 57] now gives $\Im \varphi \in h^{p}$ for all $p \in(0,1)$. Hence $\varphi \in H^{p}$ for all $p \in(0,1)$. This is a contradiction, since all functions in the Hardy spaces have bounded characteristic. Moreover, $\varphi$ clearly maps $[0,1)$ onto $[0, \infty)$, and hence, by $(2.3)$, the function $A(z)$ in (2.1) grows exponentially. In particular, $A(z)$ is of unbounded characteristic.

(3) The choice $\varphi(z)=\frac{1+z}{1-z}\left(\log \frac{1}{1-z}+1\right)^{p}, p \geq 0$, in (2.1) leads to a coefficient $A(z)$ of growth $\sigma_{\log }(A)=p$, where

$$
\sigma_{\log }(A)=\limsup _{r \rightarrow 1^{-}} \frac{\log ^{+} T(r, A)}{\log (-\log (1-r))}
$$

is the logarithmic order of $A(z)$ [7]. This calculation requires a fair amount of work, but the details are essentially worked out in [7, pp. 172-174].

(4) The choice $\varphi(z)=\left(\frac{1+z}{1-z}\right)^{p+1}, p \geq 0$, in (2.1) leads to a coefficient $A(z)$ of growth $\sigma(A)=p$. The details can be worked out easily, see [6, p. 753].

By Example 1 it seems plausible that no growth condition on a finite-order $A(z)$ alone implies (1.6). The main result below shows that it is possible to construct a function $A(z)$ analytic in $\mathbf{D}$ and of arbitrarily rapid growth such that (1.1) possesses two linearly independent solutions each having no zeros. This is a unit disc analogue of the corresponding reasoning in [3, p. 356].

Theorem 1. Let $\Lambda(r)$ be an increasing and continuous function defined on the interval $[0,1)$ such that $\Lambda^{\prime \prime}(r)>0$ and

$$
\lim _{r \rightarrow 1^{-}} \frac{\Lambda(r)}{-\log (1-r)}=\infty
$$

Then it is possible to construct a function $A(z)$ analytic in $\mathbf{D}$ of growth

$$
\lim _{r \rightarrow 1^{-}} \frac{T(r, A)}{\Lambda(r)}=\infty
$$

such that (1.1) possesses linearly independent solutions $f_{1}, f_{2}$ each having no zeros. Moreover, the product function $E=f_{1} f_{2}$ satisfies

$$
\lim _{r \rightarrow 1^{-}} \frac{T(r, E)}{\Lambda(r)}=\infty
$$

In light of cases (3) and (4) in Example 1, the assumption (2.4) does not seem too restrictive. When proving Theorem 1 in Section 4, we rely on a Linden-Shea construction $[19,25]$ on an analytic function of prescribed asymptotic growth. This 
construction depends on (2.4). It may be possible that (2.4) can be weakened to " $\Lambda(r)$ is unbounded", but this requires new results on asymptotic growth.

Remark 1. Let $b \in\left(0, e^{-6}\right)$ be an arbitrary constant. The proof of Theorem 1 shows that, in addition to (2.6), the product function $E=f_{1} f_{2}$ also satisfies

$$
\limsup _{r \rightarrow 1^{-}} \frac{T\left(r, \frac{E^{\prime}}{E}\right)}{\Lambda(1-b(1-r))} \leq 1 .
$$

We note that the constant $e^{-6}$ may not be the best possible.

We conclude that no growth condition on $A(z)$ alone yields a unit disc analogue of the BL-conjecture. This settles one open problem stated in a recent paper by Cao and $\mathrm{Yi}[4]$. Another open problem in [4] is stated as follows:

Let $A(z)$ be a non-admissible analytic function in D. Suppose that $f_{1}, f_{2}$ are two linearly independent solutions of (1.1), and set $E=f_{1} f_{2}$. It is known that in this case $\sigma(E) \leq \sigma\left(f_{1}\right)=\sigma\left(f_{2}\right)$. Can we obtain an equality instead of an inequality here?

We will demonstrate that a strict inequality $\sigma(E)<\sigma\left(f_{1}\right)=\sigma\left(f_{2}\right)$ typically holds. To begin with, recall that $f_{1}=e^{g}$ and $f_{2}=e^{g+h}$ are linearly independent solutions of (1.1), where the coefficient $A(z)$ is given by (2.1). Since $h$ denotes a primitive function of $e^{\varphi}$, and since

$$
E=f_{1} f_{2}=e^{2 g+h}=e^{-\varphi},
$$

our claim is intuitively clear. For example, suppose that $\varphi$ is an unbounded analytic function in $\mathbf{D}$ with its range in the right-half plane, say $\varphi(z)=\frac{1+z}{1-z}$. Then $E$ is bounded in $\mathbf{D}$ and $A(z)$, as defined in (2.1), is of bounded characteristic (and hence non-admissible). Meanwhile, $\sigma\left(f_{1}\right)=\sigma\left(f_{2}\right)=\infty$. Hence it is possible that the solutions $f_{1}, f_{2}$ are of infinite order of growth, while their product $E$ belongs to $H^{\infty}$. A strict inequality may hold even in the finite-order case by [13, p. 1052]. Indeed, for $\alpha \geq 0$ and for a fixed branch, the functions

$$
f_{j}(z)=(1-z)^{\frac{\alpha+2}{2}} \exp \left((-1)^{j}\left(\frac{1}{1-z}\right)^{\alpha+1}\right), \quad j=1,2,
$$

are linearly independent solutions of (1.1), where

$$
A(z)=-\frac{\alpha(\alpha+2)}{4(1-z)^{2}}-\frac{(\alpha+1)^{2}}{(1-z)^{2 \alpha+4}} .
$$

It is clear that $\sigma\left(f_{1}\right)=\sigma\left(f_{2}\right)=\alpha$, while the product function $E$ is again in $H^{\infty}$.

We note that the example involving (2.9) and (2.10) illustrates the sharpness of the main result in [16] and is more elementary than the examples in [16].

\section{Comparison of $T(r, \varphi)$ and $T\left(r, e^{\varphi}\right)$}

The function $A(z)$ in Theorem 1 will be constructed by means of (2.1). To prove Theorem 1, we need to know how $T(r, \varphi)$ and $T\left(r, e^{\varphi}\right)$ are related to each other. Referring to the paper by Clunie [8] for a parallel discussion in the case of complex plane, we believe that the discussion below is also of independent interest.

We remind the reader that $\varphi$ may be unbounded, with its range in the lefthalf plane, while $e^{\varphi} \in H^{\infty}$. The Möbius transformation $\varphi(z)=-\frac{1+z}{1-z}$ is a typical 
example of such a case. For $T\left(r, e^{\varphi}\right)$ to be unbounded, the range of $\varphi$ should have an unbounded intersection with the right half-plane. If $p>0$, then the functions $\varphi$ in cases (3) and (4) of Example 1 satisfy

$$
\sup _{z \in \mathbf{D}}\left(1-|z|^{2}\right)|\varphi(z)|=\infty .
$$

The growth rate (3.1) is, however, not necessary for $T\left(r, e^{\varphi}\right)$ to be unbounded. Indeed, let $\varphi$ be the lacunary series

$$
\varphi(z)=\sum_{n=1}^{\infty} \frac{1}{\sqrt{n}} z^{2^{n}}
$$

studied by Pommerenke [22]. The reasoning in [22, p. 694] shows that $\varphi \in \mathscr{B}$, and that $\varphi$ has radial limits almost nowhere on $\partial \mathbf{D}$. This means that $\varphi$ is of unbounded characteristic [9, p. 17], and the reasoning in Example 1(2) shows that $e^{\varphi}$ is of unbounded characteristic as well. An alternative proof for the latter claim can be achieved as follows: The function $e^{\varphi}$ has the radial limit 0 almost nowhere on $\partial \mathbf{D}$ by means of Privalov's uniqueness theorem [23, p. 325]. All other radial limits of $e^{\varphi}$ on a set of positive Lebesgue measure are prevented by the properties of $\varphi$. Hence $e^{\varphi}$ is of unbounded characteristic.

Lemma 1. Let $\varphi$ be analytic and admissible in $\mathbf{D}$. Then $e^{\varphi}$ is admissible, and

$$
\limsup _{r \rightarrow 1^{-}} \frac{T\left(r, e^{\varphi}\right)}{T(r, \varphi)}=\infty
$$

In what follows, we will be dealing with exceptional sets. In the literature they are typically handled with [2, Lemma C] due to Bank. However, in Section 4 we will make full use of the following slight generalization of Bank's lemma. The proof is an easy modification of that in [2] and is therefore omitted.

Lemma 2. Let $r_{0} \in[0,1)$, and suppose that $g(r)$ and $h(r)$ are nondecreasing functions on $\left[r_{0}, 1\right)$ such that $g(r) \leq h(r)$ for all $r \notin F$, where the set $F \subset\left[r_{0}, 1\right)$ has a finite logarithmic measure $\operatorname{lm}(F)=\int_{F} d r /(1-r)$. Set $s_{b}(r)=1-b(1-r)$ for any $b \in\left(0, e^{-\operatorname{lm}(F)}\right)$. Then $g(r) \leq h\left(s_{b}(r)\right)$ for all $r \in\left[r_{0}, 1\right)$.

Proof of Lemma 1. Let $k \in \mathbf{N}$. Using the first and the second fundamental theorems, we conclude that

$$
\begin{aligned}
(2 k-1) T(r, \varphi) & \leq \sum_{n=-k}^{k} \bar{N}\left(r, \frac{1}{\varphi-2 \pi n i}\right)+S(r, \varphi) \\
& \leq N\left(r, \frac{1}{e^{\varphi}-1}\right)+S(r, \varphi) \leq T\left(r, e^{\varphi}\right)+S(r, \varphi)
\end{aligned}
$$

outside of a possible exceptional set $F \subset[0,1)$ for which $\operatorname{lm}(F)<\infty$. Hence

$$
\limsup _{r \rightarrow 1^{-}} \frac{T\left(r, e^{\varphi}\right)}{T(r, \varphi)} \geq \limsup _{\substack{r \rightarrow 1^{-} \\ r \notin F}} \frac{T\left(r, e^{\varphi}\right)}{T(r, \varphi)} \geq 2 k-1 .
$$

Since $k \in \mathbf{N}$ can be chosen arbitrarily large, we deduce that (3.2) holds. To prove that $e^{\varphi}$ is admissible, let $b \in\left(0, e^{-\operatorname{lm}(F)}\right)$. By Lemma 2 and (3.3), we have

$$
\left(2 k-\frac{3}{2}\right) T(r, \varphi) \leq T\left(s_{b}(r), e^{\varphi}\right), \quad r \in[0,1) .
$$


Since $\log \left(1-s_{b}(r)\right)=(1+o(1)) \log (1-r)$ and $\varphi$ is admissible, it follows that $e^{\varphi}$ is admissible as well.

By letting $k \rightarrow \infty$ in (3.4), we deduce that

$$
\lim _{r \rightarrow 1^{-}} \frac{T\left(s_{b}(r), e^{\varphi}\right)}{T(r, \varphi)}=\infty .
$$

This is closely related to (3.2). We proceed to a more complicated approach that allows us to replace "lim sup" in (3.2) by "lim", or alternatively, to take $b=1$ in (3.5). We state this result as the following unit disc analogue of a well known plane result [8, Theorem 2] due to Clunie.

Lemma 3. Suppose that $\varphi$ is analytic in $\mathbf{D}$ and of unbounded characteristic. Then $e^{\varphi}$ is of unbounded characteristic, and

$$
\lim _{r \rightarrow 1^{-}} \frac{T\left(r, e^{\varphi}\right)}{T(r, \varphi)}=\infty
$$

In particular, if $\varphi$ is admissible, then $e^{\varphi}$ is admissible.

Proof. By the discussion in Example 1(2), $e^{\varphi}$ is of unbounded characteristic. Hence it remains to prove (3.6).

We require the following statement [21, p. 276]: If $f$ is meromorphic in $\mathbf{D}$ and of unbounded characteristic, then for all $a \in \mathbf{C}$ outside a set of zero capacity, depending on $f$, we have

$$
\lim _{r \rightarrow 1^{-}} \frac{N\left(r, \frac{1}{f-a}\right)}{T(r, f)}=1
$$

It is known that a point set in $\mathbf{C}$ of zero capacity cannot have a continuum as its subset, and that the set of all complex rational points in $\mathbf{C}$ forms a set of zero capacity. Further, the union of two sets of zero capacity is also of zero capacity.

Let $F \subset \mathbf{C}$ be the exceptional set of zero capacity related to the functions $\varphi$ and $e^{\varphi}$ of unbounded characteristic. Then a point $a \in \mathbf{C} \backslash\{0\}$ can be found such that the point $a$ itself and the points

$$
w_{n}=\log |a|+i(\arg (a)+2 n \pi), \quad n \in \mathbf{Z},
$$

all avoid the set $F$, for otherwise $F$ would include a continuum. For such $a$ the points $w_{n}$ form an infinite zero sequence of $e^{\varphi}-a$. Moreover,

$$
\lim _{r \rightarrow 1^{-}} \frac{N\left(r, \frac{1}{e^{\varphi}-a}\right)}{T\left(r, e^{\varphi}\right)}=1 \quad \text { and } \quad \lim _{r \rightarrow 1^{-}} \frac{N\left(r, \frac{1}{\varphi-w_{n}}\right)}{T(r, \varphi)}=1
$$

for all $n \in \mathbf{Z}$. Let $k \in \mathbf{N}$ be any constant. Then

$$
N\left(r, \frac{1}{e^{\varphi}-a}\right) \geq \sum_{n=-k}^{k} N\left(r, \frac{1}{\varphi-w_{n}}\right) .
$$

Combining (3.7) and (3.8), we conclude that

$$
\liminf _{r \rightarrow 1^{-}} \frac{T\left(r, e^{\varphi}\right)}{T(r, \varphi)} \geq 2 k+1 .
$$

Since $k$ can be chosen arbitrarily large, the assertion (3.6) follows. 


\section{Proof of Theorem 1}

We require the following result on constructing an analytic function in $\mathbf{D}$ with a prescribed asymptotic growth.

Theorem 2 ([19, 25]). Let $\Lambda(r)$ be an increasing, convex and continuous function defined on the interval $[0,1)$ such that $(2.4)$ holds. Then there exists a function $f$ analytic in $\mathbf{D}$ such that $T(r, f) \sim \Lambda(r)$ as $r \rightarrow 1^{-}$.

Remark 2. An exceptional set $F \subset[0,1)$ typically appears after using either the lemma on the logarithmic derivative (LLD) or the second main theorem (SMT). In both cases $\operatorname{lm}(F)$ is uniformly bounded from above. This can be seen by choosing $\phi(r)=1-r$ and $\psi(r)=\log ^{2} r$ in [5, Theorems 3.4.1 and 4.2.1]. More precisely, let $f$ be meromorphic in $\mathbf{D}$ and of unbounded characteristic, and let $r_{0} \in[0,1)$ be such that $T\left(r_{0}, f\right) \geq e$. Then the inequalities in LLD and SMT hold for $r \notin\left[0, r_{0}\right] \cup F$, where $F \subset\left[r_{0}, 1\right)$ satisfies $\operatorname{lm}(F) \leq 2$. Hence Lemma 2 would be applicable for any choice of $b \in\left(0, e^{-2}\right)$.

We will make use of this reasoning. Due to (2.4), there exists a constant $r_{0} \in[0,1)$ such that $\Lambda\left(r_{0}\right) \geq e$. In what follows, the constant $r_{0}$ may not be the same each time it occurs. Set $s_{b}(r)=1-b(1-r)$ for $b \in\left(0, e^{-4}\right)$. Define $\Lambda_{0}(r)=\Lambda\left(s_{b}(r)\right)$. Clearly $\Lambda_{0}(r)$ is increasing and continuous on $[0,1)$, and satisfies

$$
\lim _{r \rightarrow 1^{-}} \frac{\Lambda_{0}(r)}{-\log (1-r)} \geq \lim _{r \rightarrow 1^{-}} \frac{\Lambda\left(s_{b}(r)\right)}{-\log \left(1-s_{b}(r)\right)}\left(1+\frac{4}{-\log (1-r)}\right)=\infty .
$$

Moreover, $\Lambda_{0}^{\prime \prime}(r)=\Lambda^{\prime \prime}\left(s_{b}(r)\right) b^{2}>0$, so that $\Lambda_{0}(r)$ is convex. By Theorem 2 there exists a function $\varphi$ analytic in $\mathbf{D}$ such that $T(r, \varphi) \sim \Lambda_{0}(r)$ as $r \rightarrow 1^{-}$. For this particular $\varphi$, define $A(z)$ as in (2.1). Clearly $\varphi$ is admissible, and hence by $[5$, Theorem 3.4.1] and Remark 2, we have

$$
T\left(r, \varphi^{(j)}\right)=m\left(r, \varphi^{(j)}\right) \leq m(r, \varphi)+m\left(r, \frac{\varphi^{(j)}}{\varphi}\right)=(1+o(1)) T(r, \varphi)
$$

for all $r \in\left[r_{0}, 1\right)$ outside of respective exceptional sets $F_{j} \subset\left[r_{0}, 1\right)$ satisfying $\operatorname{lm}\left(F_{j}\right) \leq$ 2 for $j=1,2$. Denote $F=F_{1} \cup F_{2}$, so that $\operatorname{lm}(F) \leq 4$. Combining (2.1) and (4.1) with Lemma 3, we conclude that

$$
T(r, A) \geq \frac{3}{2} T\left(r, e^{\varphi}\right), \quad r \notin\left[0, r_{0}\right) \cup F .
$$

By Lemma 2, we have

$$
\frac{T\left(s_{b}(r), A\right)}{\Lambda\left(s_{b}(r)\right)} \geq \frac{3}{2} \cdot \frac{T\left(r, e^{\varphi}\right)}{T(r, \varphi)} \cdot \frac{T(r, \varphi)}{\Lambda_{0}(r)} \geq \frac{T\left(r, e^{\varphi}\right)}{T(r, \varphi)}, \quad r \in\left[r_{0}, 1\right) .
$$

The assertion (2.5) now follows by means of (4.2) and Lemma 3. Using (2.8), we deduce that

$$
\frac{T(r, E)}{\Lambda(r)}=\frac{T\left(r, e^{\varphi}\right)}{T(r, \varphi)} \cdot \frac{T(r, \varphi)}{\Lambda_{0}(r)} \cdot \frac{\Lambda_{0}(r)}{\Lambda(r)} \geq \frac{T\left(r, e^{\varphi}\right)}{T(r, \varphi)} \cdot \frac{T(r, \varphi)}{\Lambda_{0}(r)} .
$$

The assertion (2.6) now follows by Lemma 3.

Proof of (2.7). We note that the formulas (2.8) and (4.1) yield

$$
T\left(r, \frac{E^{\prime}}{E}\right)=T\left(r, \varphi^{\prime}\right)=(1+o(1)) T(r, \varphi) \sim(1+o(1)) \Lambda_{0}(r) .
$$


To see that (2.7) holds, it remains to use Lemma 2 in the case $\operatorname{lm}(F) \leq 2$.

\section{Concluding remarks on zero-free solution bases}

The solutions $f_{1}, f_{2}$ in $(2.9)$ are of the form

$$
f_{j}(z)=\exp \left(g(z)+(-1)^{j} h(z)\right), \quad j=1,2,
$$

where $g$ and $h$ are analytic in $\mathbf{D}$. Indeed, by choosing $g(z)=\frac{\alpha+2}{2} \log (1-z)$ and $h(z)=(1-z)^{-\alpha-1}$, we see that (5.1) reduces to (2.9). In the general case, for $f_{1}, f_{2}$ in (5.1) to be linearly independent solutions of (1.1), the function $h$ must be nonconstant. Moreover, by substituting $f_{1}, f_{2}$ in (1.1), we get, after a simplification, that the functions $g, h$ depend on each other by the equation

$$
\frac{h^{\prime \prime}}{h^{\prime}}=-2 g^{\prime} \text {. }
$$

Hence $h^{\prime}=C e^{-2 g}$ for some $C \in \mathbf{C} \backslash\{0\}$. The product function $E=f_{1} f_{2}=e^{2 g}$ and the ratio $G=f_{2} / f_{1}=e^{2 h}$ are now easy to deal with. Recall that $S_{G}=2 A$, where

$$
S_{G}=\left(\frac{G^{\prime \prime}}{G^{\prime}}\right)^{\prime}-\frac{1}{2}\left(\frac{G^{\prime \prime}}{G^{\prime}}\right)^{2}
$$

is the Schwarzian derivative of $G$. Since $G=e^{2 h}$, an easy computation yields

$$
2 A=S_{h}-2\left(h^{\prime}\right)^{2} \text {. }
$$

In particular, if $h$ is a (constant times) Möbius transformation, then $S_{h}=0$, and so $A=-\left(h^{\prime}\right)^{2}$. This is the theory behind [13, Example 5.3], for example. In the general case, using (5.2), we may write (5.3) alternatively as

$$
A=-g^{\prime \prime}-\left(g^{\prime}\right)^{2}-\left(h^{\prime}\right)^{2} .
$$

Hence a zero-free solution base as well as the coefficient $A(z)$ of (1.1) can be written in terms of two parameter functions $g, h$ by means of (5.1) and (5.4).

We note that the solutions $f_{1}, f_{2}$ in $(5.1)$ can also be written in terms of the BL-method described in Section 2, and hence this approach is not new. However, sometimes this approach is easier to use in constructing examples of zero-free solution bases. Suitable computer software is also useful.

Corresponding to the reasoning in the beginning of Section 2, we have the following claim: If $g \in \mathscr{B}$, then the solutions $f_{1}, f_{2}$ in (5.1) are of finite order. To prove this, we first observe that $h^{\prime}=C e^{-2 g}$ is an $\mathscr{H}$-function by (2.2). Since

$$
|h(z)| \leq|h(0)|+\int_{0}^{|z|}\left|h^{\prime}(\zeta)\right||d \zeta|
$$

it follows that $h$ is also an $\mathscr{H}$-function. Let $\sigma_{M}(f)$ be the maximum modulus order of a function $f$ analytic in D. The sharp estimates $\sigma(f) \leq \sigma_{M}(f) \leq \sigma(f)+1$ are well-known. We now conclude that $\sigma\left(f_{j}\right) \leq \sigma_{M}\left(f_{j}\right)<\infty$ for $j=1,2[16]$.

In a private communication, Gröhn (University of Eastern Finland) pointed out that if

$$
\left(1-|z|^{2}\right)\left|g^{\prime}(z)\right| \leq 1 / 2, \quad z \in \mathbf{D}
$$

then $h$ is univalent in $\mathbf{D}$. This claim follows by applying [23, p. 172] on (5.2).

The discussion above applies in the case of $\mathbf{C}$ as well. Indeed, if $g$ is a polynomial, then $\rho(h)=\operatorname{deg}(g)=\rho(A)$ by $h^{\prime}=C e^{-2 g}$ and (5.4). If $h$ in turn is a polynomial, 
then so is $h^{\prime}$, and hence $g$ must be a constant function. In this case $\operatorname{deg}(h)=1$ and $A(z)$ is a constant function. The latter case is the only possibility for constructing entire solutions of finite order. If $g$ is transcendental entire, then so is $h$, and $A(z)$ in (5.4) is of infinite order of growth by [8, Theorem 2].

We note that if the starting point is a non-vanishing analytic function $E$ (hence a BL-function), then the method due to Shen in [26] can also be used in constructing zero-free solution bases. Indeed, it turns out that $E$ is then a product of two linearly independent zero-free solutions. This method is originally written in the case of $\mathbf{C}$, but it is clearly valid in the case of $\mathbf{D}$ as well. For example, the choice $E(z)=\frac{(1-z)^{\alpha+2}}{2(\alpha+1)}$ for a fixed $\alpha \geq 0$ in [26] yields

$$
A(z)=-\frac{1}{4}\left(\frac{1}{E(z)^{2}}+2 \frac{E^{\prime \prime}(z)}{E(z)}-\left(\frac{E^{\prime}(z)}{E(z)}\right)\right)=-\frac{\alpha(\alpha+2)}{4(1-z)^{2}}-\frac{(\alpha+1)^{2}}{(1-z)^{2 \alpha+4}}
$$

as in (2.10). The functions in the corresponding zero-free solution base

$$
g_{j}(z)=E(z)^{\frac{1}{2}} \exp \left(\frac{(-1)^{j}}{2} \int_{0}^{z} \frac{d \zeta}{E(\zeta)}\right), \quad j=1,2,
$$

then reduce to constant multiples of the functions $f_{1}, f_{2}$ in $(2.9)$, respectively.

We have seen that it is possible to construct zero-free solutions bases $\left\{f_{1}, f_{2}\right\}$ for (1.1) in the cases of $\mathbf{C}$ and $\mathbf{D}$. It has recently been proved [14] in both cases that arbitrary linear combinations $f=C_{1} f_{1}+C_{2} f_{2}$ typically have the maximal quantity of zeros when compared to the growth of $f_{1}, f_{2}$.

\section{References}

[1] Anderson, J. M., J. Clunie, and Ch. Pommerenke: On Bloch functions and normal functions. - J. Reine Angew. Math. 270, 1974, 12-37.

[2] BANK, S.: A general theorem concerning the growth of solutions of first-order algebraic differential equations. - Compositio Math. 25:1, 1972, 61-70.

[3] Bank, S., and I. Laine: On the oscillation theory of $f^{\prime \prime}+A f=0$ where $A$ is entire. Trans. Amer. Math. Soc. 273:1, 1982, 351-363.

[4] CAO, T.-B., and H.-X. Yı: On the complex oscillation theory of $f^{\prime \prime}+A(z) f=0$ where $A(z)$ is analytic in the unit disc. - Math. Nachr. 282:6, 2009, 820-831.

[5] Cherry, W., and Z. YE: Nevanlinna's theory of value distribution. The second main theorem and its error terms. - Springer Monogr. Math., Springer-Verlag, Berlin, 2001.

[6] Chyzhykov, I., G. G. Gundersen, and J. Heittokangas: Linear differential equations and logarithmic derivative estimates. - Proc. London Math. Soc. 86:3, 2003, 735-754.

[7] Chyzhykov, I., J. Heittokangas, and J. RÄтtÿ̈: Finiteness of $\varphi$-order of solutions of linear differential equations in the unit disc. - J. Anal. Math. 109:1, 2009, 163-198.

[8] Clunie, J.: The composition of entire and meromorphic functions. - Mathematical Essays Dedicated to A. J. McIntyre, Ohio Univ. Press, 1970, 75-92.

[9] Duren, P.: Theory of $H^{p}$ spaces. - Academic Press, New York-San Francisco-London, 1970.

[10] Hayman, W.: On Nevanlinna's second theorem and extensions. - Rend. Circ. Mat. Palermo, Ser. II, 1952, 346-392.

[11] Hayman, W.: Meromorphic functions. - Oxford Math. Monogr., Clarendon Press, Oxford, 1964.

[12] Heittokangas, J.: On complex differential equations in the unit disc. - Ann. Acad. Sci. Fenn. Math. Diss. 122, 2000, 1-54. 
[13] Heittokangas, J., R. Korhonen, and J. Rättyä: Linear differential equations with coefficients in weighted Bergman and Hardy spaces. - Trans. Amer. Math. Soc. 360:2, 2008, $1035-1055$.

[14] Heittokangas, J., and J. Räтtyä: Zero distribution of solutions of complex linear differential equations determines growth of coefficients. - Math. Nachr. (to appear).

[15] Korenblum, B.: An extension of the Nevanlinna theory. - Acta Math. 135:3-4, (1975), 187219.

[16] Korhonen, R., and J. RÄttyä: Linear differential equations in the unit disc with analytic solutions of finite order. - Proc. Amer. Math. Soc. 135, 2007, 1355-1363.

[17] Laine, I.: Nevanlinna theory and complex differential equations. - Walter de Gruyter, BerlinNew York, 1993.

[18] Laine, I., and K. TohgE: The Bank-Laine conjecture - a survey. - Some topics on value distribution and differentiability in complex and $p$-adic analysis, edited by A. Escassut, W. Tutschke and C. C. Yang, Science Press, Beijing, 398-417.

[19] Linden, C.: Functions analytic in a disc having prescribed asymptotic growth properties. J. London Math. Soc. 2:2, 1970, 267-272.

[20] Littlewood, J. E.: Lectures on the theory of functions. - Oxford Univ. Press, 1944.

[21] Nevanlinna, R.: Analytic functions. - Grundlehren Math. Wiss. 162, Springer-Verlag, New York-Berlin, 1970.

[22] Pommerenke, Ch.: On Bloch functions. - J. London Math. Soc. 2:2, 1970, 689-695.

[23] Pommerenke, Ch.: Univalent functions. - Studia Mathematica/Mathematische Lehrbücher, Band XXV. Vandenhoeck \& Ruprecht, Göttingen, 1975.

[24] Rossi, J.: Second order differential equations with transcendental coefficients. - Proc. Amer. Math. Soc. 97:1, 1986, 61-66.

[25] SHEA, D. F.: Functions analytic in a finite disk and having asymptotically prescribed characteristic. - Pacific J. Math. 17, 1966, 549-560.

[26] Shen, L.-C.: Construction of a differential equation $y^{\prime \prime}+A y=0$ with solutions having the prescribed zeros. - Proc. Amer. Math. Soc. 95, 1985, 544-546.

[27] SHen, L.-C.: Solution to a problem of S. Bank regarding exponent of convergence of zeros of the solutions of differential equation $f^{\prime \prime}+A f=0$. - Kexue Tongbao (English ed.) 30:12, 1985, $1579-1585$.

Received 31 August 2010 